\title{
Pearls and pitfalls of posterior superior iliac spine reference frame placement for spinal navigation: cadaveric series
}

\author{
*Brendan F. Judy, MD, ${ }^{1}$ Hector Soriano-Baron, MD, ${ }^{1}$ Yike Jin, MD, ${ }_{1}^{1}$ Hesham M. Zakaria, MD, ${ }^{1}$ Srujan Kopparapu, BS, BA, ${ }^{1}$ \\ Mir Hussain, BS, ${ }^{2}$ Connor Pratt, BS, ${ }^{2}$ and Nicholas Theodore, $\mathrm{MD}^{1}$ \\ ${ }^{1}$ Department of Neurosurgery, Johns Hopkins Hospital, Baltimore, Maryland; and ${ }^{2}$ Globus Medical, Audobon, Pennsylvania
}

BACKGROUND Navigation and robotics are important tools in the spine surgeon's armamentarium and use of these tools requires placement of a reference frame. The posterior superior iliac spine (PSIS) is a commonly used site for reference frame placement, due to its location away from the surgical corridor and its ability to provide solid fixation. Placement of a reference frame requires not only familiarity with proper technique, but also command of the relevant anatomy.

OBSERVATIONS Cadaveric analysis demonstrates a significant difference in PSIS location in males versus females, and additionally provides average thickness for accurate placement.

LESSONS In this technical note, the authors describe the precise technique for PSIS frame placement in addition to relevant anatomy and offer solutions to commonly encountered problems.

https://thejns.org/doi/abs/10.3171/CASE21621

KEYWORDS navigation; robotic surgery; spine surgery

The use of neuronavigation has become increasingly prevalent in spine surgery for accurate placement of spinal instrumentation. ${ }^{1}$ Many growing fields such as robotic spine surgery and other minimally invasive spine surgical techniques rely heavily on intraoperative registration and neuronavigation for proper instrumentation placement, particularly in situations in which direct visualization of anatomy is not possible. Robotic spine surgery with neuronavigation in particular has been shown to have favorable accuracy rates, ${ }^{2,3}$ decreased blood loss, and shorter hospital length of stay. ${ }^{4}$ Proper reference frame placement is paramount to achieving the level of navigational accuracy needed to perform these surgeries safely and efficiently. Multiple factors influence placement of the reference frame, including patient size, bone quality, gender, and skin elasticity. If the reference frame is placed incorrectly, registration failure and navigation deviation can occur, resulting in prolonged surgical and anesthesia time, unacceptable screw placement, and ultimately patient harm. Hartl et al. ${ }^{5}$ in a worldwide survey demonstrated that the perceived increase in operative time associated with using spinal navigation was a significant stated reason for its lack of use. Therefore, accurate initial placement of the reference frame is crucial for not only ensuring accuracy but also minimizing operative delay. The posterior superior iliac spine (PSIS) has been shown to be an excellent location for reference frame placement due to its location outside of the typical surgical corridor and the ability to provide solid bony purchase. ${ }^{6}$ In this technical note, we discuss the technique of reference frame placement in the PSIS, highlighting the pearls and pitfalls of placement to ensure success.

\section{Study Description}

Computed tomography scans of 21 cadavers, 7 females and 14 males, were analyzed using Globus proprietary software (Globus Medical). The cadaveric measurements were analyzed using Microsoft Excel (Microsoft). Continuous variables were analyzed using paired and unpaired t-tests. 

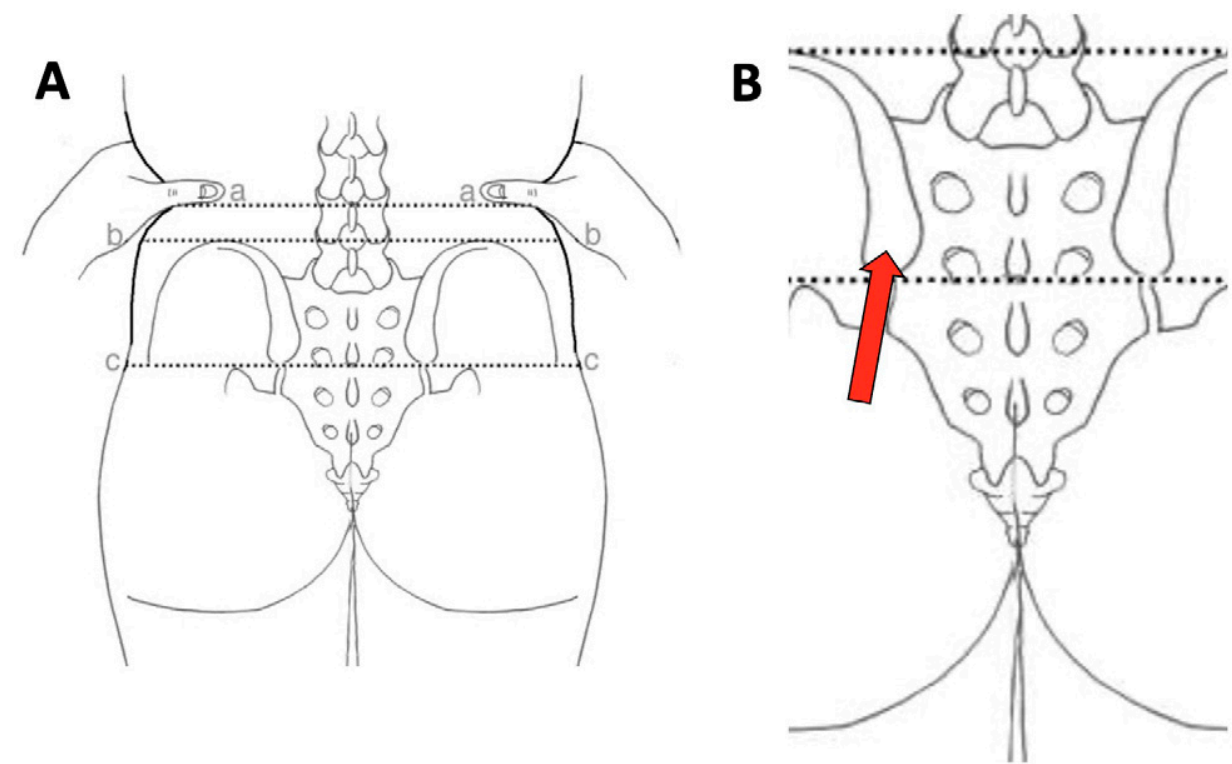

FIG. 1. A: Line $b$ represents the intercristal line, which approximates the L4-5 disc space. Line $c$ indicates the PSIS and S2 foramen. B: The reference frame is inserted into the PSIS (red arrow). Image used with permission from Dr. Robin Chakraverty/Wiley Publishing.

\section{Technique \\ Palpation}

Placement of the reference frame in the PSIS utilizes anatomical landmarks (Fig. 1). The PSIS is located lateral to the midline spinous processes, and a review of 100 computed tomography scans ${ }^{7}$ showed that the craniocaudal location of the PSIS correlated $81 \%$ of the time with that of the S2 spinous process. The PSIS may be identified by first finding the more commonly palpated intercristal line (also known as Jacoby line or Tuffier line; this line intersects the most superior portion of the iliac crests) that approximates the L4-5 intervertebral space. Starting with both hands on the intercristal line, one can trace the iliac crests inferiorly and medially with the thumbs. This leads to the PSIS caudally, which can then be palpated and marked accordingly (Video 1).

\section{VIDEO 1. Clip showing palpation of PSIS. Click here to view.}

\section{Approximation}

Cadaveric analysis revealed a mean distance off midline of 5.09 $\mathrm{cm}$ with a statistically significant difference in female specimens compared with male specimens $(5.54 \mathrm{~cm}$ versus $4.86 \mathrm{~cm}$, respectively; $p<0.001$ ) (Fig. 2). The mean thickness of the PSIS in the anteroposterior orientation was $2.08 \mathrm{~cm}$ and the PSIS was $3.72 \mathrm{~cm}$ superior to the sacroiliac joint in the craniocaudal orientation.

\section{Placement}

Once the PSIS is identified, a $1 \mathrm{~cm}$ vertical skin incision is made approximately $2 \mathrm{~cm}$ inferior to the PSIS. The vertical incision accounts for skin elasticity and prevents tension on the reference frame post. An electrocautery device is used to control bleeding and extend the incision to the bone surface. Local anesthetic is liberally injected into the periosteum and surrounding soft tissues. A blunt instrument (e.g., a Penfield 4) is then used to palpate the boundaries of the PSIS and serves as a surrogate for placement, which is particularly helpful in patients who are obese. The reference frame post is then inserted into the incision and directed $0^{\circ}$ mediolaterally and $30^{\circ}$ to $40^{\circ}$ angled toward the feet (Fig. 3). This angle directs the reference frame away from the surgical corridor, preventing inadvertent movement of the frame intraoperatively, and the initial incision is made inferior to the PSIS to accommodate this angle while avoiding stretching of the skin. A mallet is used to insert the post into the PSIS approximately 1 to $2 \mathrm{~cm}$ (Fig. 4). Once the frame post is placed, its stability must be verified by applying light pressure on the base to ensure that the bony purchase is adequate to prevent movement. The reference frame should then be

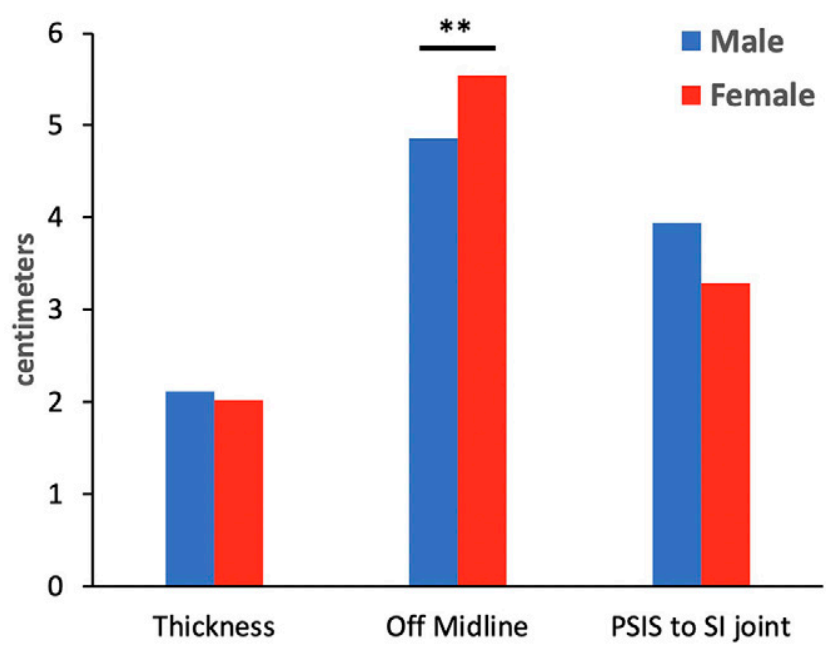

FIG. 2. The mean distance off midline, thickness, and distance from the sacroiliac joint reported from 21 cadavers. $* *$ represents $p$ value $<0.001$. 


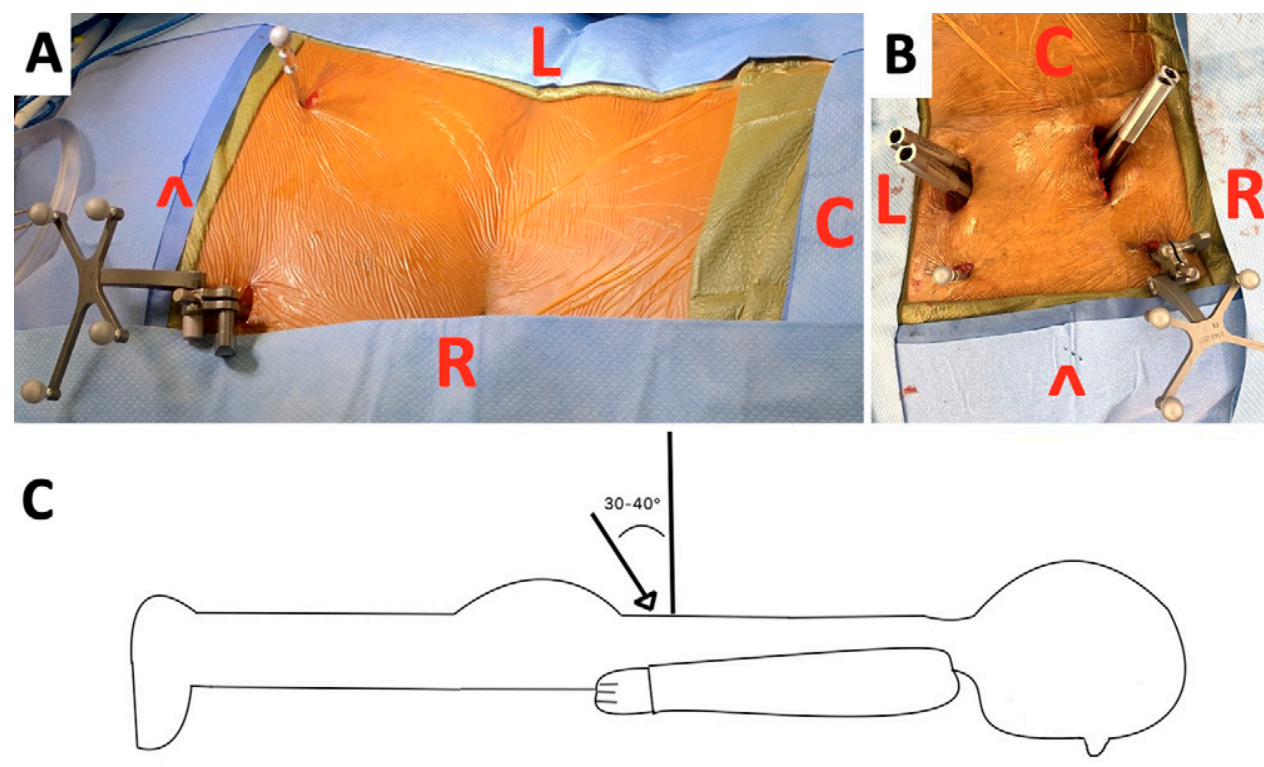

FIG. 3. A and B: Dynamic reference array placed in PSIS on right side of patient (prone position) and surveillance frame in left PSIS. Right $(R)$ and left $(L)$ of patient are labeled accordingly. Lumbar minimally invasive towers (B) with stabilizing cuffs are used for rod placement. C: Illustration of patient in prone position. The PSIS reference marker is placed at a 30- to 40-degree angle toward the feet to avoid the surgical corridor. $\mathrm{C}=$ cephalad; $\wedge$ = caudal.

positioned on the post such that it is close to the skin but not touching or depressing the skin surface. All fiducials need to be visible and away from sterile drapes to ensure navigation registration and integrity. Depending on the navigation system, a second surveillance frame may be placed on the contralateral PSIS as an additional source of navigational accuracy (Fig. 3). Anteroposterior and lateral radiographs are then obtained to sync the radiograph with the preoperative computed tomography for registration of navigation (Fig. 5); these images may be used to confirm appropriate placement of reference frame.

The incision is closed using deep subcutaneous sutures in addition to a superficial subcutaneous layer. Glue or staples may be used to close the skin.

\section{Pearls and Pitfalls}

It can be challenging to palpate anatomical landmarks in obese patients, however, radiographs or C-arm can be used to confirm location in such patients. In addition, a longer reference frame post may be necessary to obtain adequate bony purchase while still ensuring sufficient post extends above the fat and soft tissue to attach the reference frame itself. It is essential to dissect fat and soft tissue away in the intended angle of post placement to avoid having any skin or soft tissue place pressure on the reference frame, which can lead to movement of the post and subsequent loss of registration accuracy. If poor bony purchase remains an issue and the previous techniques mentioned have already been attempted, further salvage techniques include driving the post farther into the bone and finally using the other PSIS. If the post cannot be placed, placement of an interspinous clamp is an alternative. For patients with a high sacral slope, the reference frame can prevent the steep trajectory of the robotic arm and may need to be adjusted to point more inferiorly. For placement of S2-alar-iliac screws, the reference frame may need to be angled laterally to prevent intersecting the screw trajectory. Finally, there is a slight yet statistically significant difference in male versus female PSIS location that should be noted and considered when placing the reference frame post.

In terms of pitfalls, avoiding bicortical purchase may help prevent pelvic organ injury. Avoiding medial placement, meanwhile, can prevent poor bony purchase. The middle cluneal nerve is located medially, and the superior cluneal nerves are located on the superior iliac crest laterally. ${ }^{8}$ Inferiorly and laterally, the superior gluteal
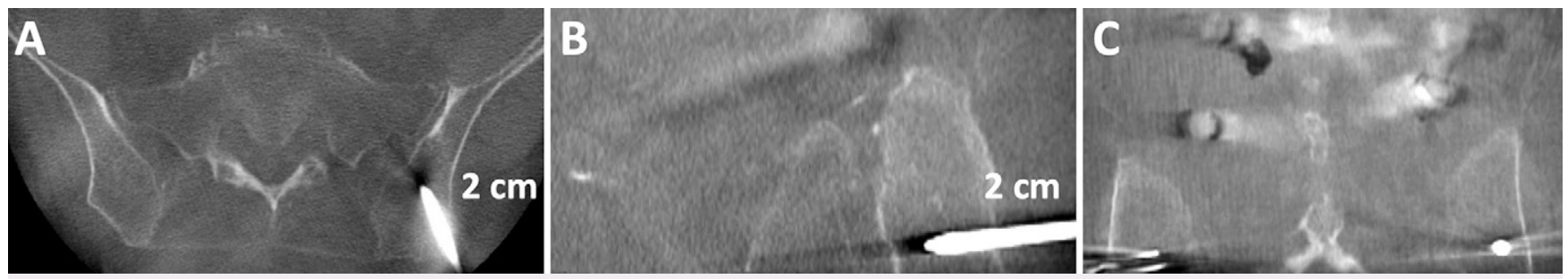

FIG. 4. O-arm images demonstrate left PSIS marker inserted approximately $2 \mathrm{~cm}$ in the axial (A), sagittal (B), and anteroposterior (C) views. 

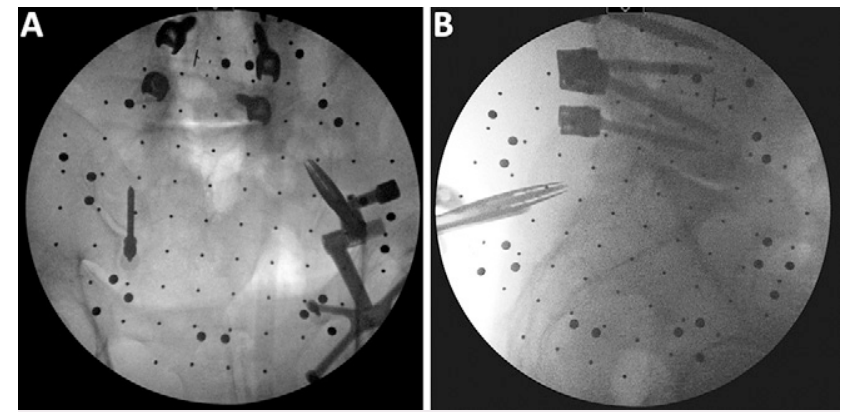

FIG. 5. Reference frame placed in PSIS on right side of patient (prone position) and surveillance frame in left PSIS in anteroposterior $(\mathbf{A})$ and lateral radiographs $(\mathbf{B})$. This patient had undergone prior lumbar fusion.

vessels are located in the superior portion of the sciatic notch. The inferior portion of the notch contains the sciatic nerve, inferior gluteal nerve and vessels in addition to the internal pudendal vessels and nerve. ${ }^{8}$ The bone thins laterally along the iliac crest, and bicortical placement in this region can injure the psoas muscle. If bleeding is encountered during placement of PSIS, it is most often bony bleeding that will stop with irrigation and time. If the bleeding is excessive and does not stop with irrigation and compression, it is possible that an artery has been injured. In this situation, the case should be stopped to obtain vascular imaging and/or vascular surgery consult. Likewise, if there is concern that an abdominal injury has occurred due to bleeding or hemodynamic changes, the surgery should be halted and general surgery should be notified immediately. If the surgeon is concerned with navigation integrity, the first step is to perform a landmark verification. This can be done by placing the handheld wand on a known landmark (e.g., L4 lamina) to see if the navigation correlates with known anatomical landmarks. If the navigation is noted to be inaccurate, the navigation must be reregistered immediately. ${ }^{9}$ Finally, issues such as wound healing and infection are rare with Best et al. ${ }^{6}$ reporting no complications with healing of PSIS incision sites in 272 patients.

\section{Discussion}

\section{Observations}

Our cadaveric analysis demonstrates a significant difference in PSIS location in males versus females, and additionally provides average thickness for accurate placement. This difference in PSIS location off the midline has not been previously reported. The use of imageguided navigation and robotic-assisted spine surgery are increasing rapidly, and correct placement of the reference frame for navigation is essential for proper instrumentation. For lumbosacral surgical procedures, the PSIS is the preferred location for the reference frame, as this location avoids obstruction of instrumentation trajectory, prevents blocking the visualization of the surgical corridor, evades streak artifact, and provides enhanced stability of the reference frame..$^{6,10}$

\section{Lessons}

Combining anatomical landmarks, palpation, and radiographic techniques, the PSIS can be easily accessed for reference frame placement with solid bony purchase and avoidance of the surgical corridor. This is a well-tolerated technique that can be performed safely and efficiently. The main limitations of this study are the small sample size
(21 cadavers) and the unknown clinical significance of the gender difference in PSIS location. This study contributes to the current literature on navigation in spine surgery with the goal of increasing patient safety and improving navigation accuracy and precision.

\section{References}

1. Tajsic T, Patel K, Farmer R, Mannion RJ, Trivedi RA. Spinal navigation for minimally invasive thoracic and lumbosacral spine fixation: implications for radiation exposure, operative time, and accuracy of pedicle screw placement. Eur Spine J. 2018;27(8):1918-1924.

2. Jiang B, Pennington Z, Zhu A, et al. Three-dimensional assessment of robot-assisted pedicle screw placement accuracy and instrumentation reliability based on a preplanned trajectory. J Neurosurg Spine. 2020;33(4):1-10.

3. Fatima N, Massaad E, Hadzipasic M, Shankar GM, Shin JH. Safety and accuracy of robot-assisted placement of pedicle screws compared to conventional free-hand technique: a systematic review and meta-analysis. Spine J. 2021; 21(2):181-192.

4. Jiang B, Pennington Z, Azad T, et al. Robot-assisted versus freehand instrumentation in short-segment lumbar fusion: Experience with real-time image-guided spinal robot. World Neurosurg. 2020;136:e635-e645.

5. Härtl R, Lam KS, Wang J, Korge A, Kandziora F, Audigé L. Worldwide survey on the use of navigation in spine surgery. World Neurosurg. 2013;79(1):162-172.

6. Best NM, Sasso RC, Garrido BJ. Computer-assisted spinal navigation using a percutaneous dynamic reference frame for posterior fusions of the lumbar spine. Am J Orthop. 2009;38(8):387-391.

7. McGaugh JM, Brismée JM, Dedrick GS, Jones EA, Sizer PS. Comparing the anatomical consistency of the posterior superior iliac spine to the iliac crest as reference landmarks for the lumbopelvic spine: a retrospective radiological study. Clin Anat. 2007;20(7): 819-825.

8. Sittitavornwong S, Falconer DS, Shah R, Brown N, Tubbs RS. Anatomic considerations for posterior iliac crest bone procurement. J Oral Maxillofac Surg. 2013;71(10):1777-1788.

9. Crawford N, Johnson N, Theodore N. Ensuring navigation integrity using robotics in spine surgery. J Robot Surg. 2020;14(1):177-183.

10. Lin HH, Lu YH, Chou PH, Chang MC, Wang ST, Liu CL. Is bony attachment necessary for dynamic reference frame in navigationassisted minimally invasive lumbar spine fusion surgery? Comput Assist Surg (Abingdon). 2019;24(1):7-12.

\section{Disclosures}

Mr. Pratt and Mr. Hussain are employees of Globus Medical. Dr. Theodore reports personal fees from Globus Medical outside the submitted work; in addition, Dr. Theodore has a patent for a robotic platform for spinal surgery with Globus Medical. No other disclosures to report.

\section{Author Contributions}

Conception and design: Theodore, Judy, Soriano-Baron, Zakaria. Acquisition of data: Judy, Soriano-Baron, Zakaria, Hussain. Analysis and interpretation of data: Judy, Soriano-Baron, Zakaria, Kopparapu, Hussain. Drafting the article: Judy, Soriano-Baron, Jin, Zakaria, Kopparapu, Hussain. Critically revising the article: Theodore, Judy, Soriano-Baron, Jin, Zakaria, Hussain. Reviewed submitted version of manuscript: Judy, Jin, Kopparapu, Hussain, Pratt. Statistical analysis: Judy. Administrative/technical/material support: Theodore. Study supervision: Theodore.

\section{Supplemental Information \\ Videos}

Video 1. https://vimeo.com/658994652. 
Previous Presentations

Portions of this work were presented as a digital poster at the 2021

Congress of Neurological Surgeons Annual Meeting, Austin, TX,

October 16-18, 2021.

\section{Correspondence}

Nicholas Theodore: Johns Hopkins University School of Medicine, Baltimore, MD. theodore@jhmi.edu. 